\title{
IDEALS AND FINITENESS CONDITIONS FOR SUBSEMIGROUPS
}

\author{
R. GRAY \\ School of Mathematics, University of East Anglia, Norwich NR4 7TJ, England \\ e-mail: Robert.D.Gray@uea.ac.uk
}

\author{
V. MALTCEV, J. D. MITCHELL and N. RUŠKUC \\ School of Mathematics and Statistics, University of St Andrews, \\ St Andrews KY16 9SS, Scotland, United Kingdom \\ e-mail: \{victor,jamesm,nik\}@mcs.st-and.ac.uk
}

(Received 30 April 2012; revised 28 November 2012; accepted 23 January 2013; first published online 13 August 2013)

\begin{abstract}
In this paper we consider a number of finiteness conditions for semigroups related to their ideal structure, and ask whether such conditions are preserved by sub- or supersemigroups with finite Rees or Green index. Specific properties under consideration include stability, $\mathcal{D}=\mathcal{J}$ and minimal conditions on ideals.
\end{abstract}

2010 Mathematics Subject Classification. 20M05, $20 \mathrm{M} 12$.

1. Introduction. Significant information about a semigroup may be obtained by studying its ideal structure and various finiteness conditions related to it. Examples include the existence of minimal ideals, stability and the property of Green's relations $\mathcal{J}$ and $\mathcal{D}$ coinciding. Such properties have been identified and investigated because of their usefulness in the study of finite semigroups; see [22, Appendix A.2]. This has led to instances where theorems that were originally proved for finite semigroups have been extended to apply to wider classes.

Our main interest here is in the study of infinite semigroups satisfying such finiteness properties relating to their ideal structure. In this context, it is natural to ask, given a semigroup satisfying a certain property, to what extent it can be changed while still continuing to satisfy the property. For example, an obvious basic question is whether the property in question is preserved under operations such as adjoining an identity element, or a zero element. Taking this one step further, one can consider this behaviour under finite changes in a number of elements. This leads to the notion of Rees index. The Rees index of a subsemigroup $T$ of semigroup $S$ is defined simply as the cardinality of the complement $S \backslash T$. Rees index was originally introduced and investigated by Jura [14-16]. Since then the theory has been developed and extended considerably, with results about Rees index appearing in different papers $[5,12,17,20,23,24,26,27]$.

Although natural, this notion is very restrictive, and as such limits the applicability of results about Rees index. For instance, it is not hard to see that an infinite group cannot have any proper subgroups of finite Rees index. Recently, in Gray and Ruškuc [10], a new approach was proposed, encompassing both Rees index and group-theoretic index, which is at the same time natural and strong enough to enable one to prove 
Table 1. Summary of Green index results

\begin{tabular}{|c|c|c|c|}
\hline & & $\begin{array}{c}\text { Finite Green } \\
\text { index subsemigroups }\end{array}$ & $\begin{array}{c}\text { Finite Green } \\
\text { index extensions }\end{array}$ \\
\hline \multirow[t]{2}{*}{$\mathcal{J}=\mathcal{D}$} & $T$ arbitrary & $\begin{array}{c}\mathrm{x} \\
\text { (Example 4.6) }\end{array}$ & $\begin{array}{c}\checkmark \\
\text { (Theorem 4.1) }\end{array}$ \\
\hline & $T$ regular & $\begin{array}{c}\checkmark \\
\text { (Theorem 4.7) }\end{array}$ & $\begin{array}{c}\checkmark \\
\text { (Theorem 4.1) }\end{array}$ \\
\hline Stability & & $\begin{array}{c}\checkmark \\
\text { (Theorem 3.2) }\end{array}$ & $\begin{array}{c}\checkmark \\
\text { (Theorem 3.2) }\end{array}$ \\
\hline Finitely many ideals & & $\begin{array}{c}\checkmark \\
\text { (Theorem 5.1) }\end{array}$ & $\begin{array}{c}\checkmark \\
\text { (Theorem 5.1) }\end{array}$ \\
\hline $\min _{R}$ & & $\begin{array}{c}\checkmark \\
\text { (Theorem 6.1) }\end{array}$ & $\begin{array}{c}\checkmark \\
\text { (Theorem 6.1) }\end{array}$ \\
\hline $\min _{J}$ & & $\begin{array}{c}\checkmark \\
\text { (Theorem 6.4) }\end{array}$ & $\begin{array}{c}\checkmark \\
\text { (Theorem 6.4) }\end{array}$ \\
\hline Global torsion & & $\begin{array}{c}\checkmark \\
\text { (Theorem 8.1) }\end{array}$ & $\begin{array}{c}\checkmark \\
\text { (Theorem 8.1) }\end{array}$ \\
\hline Eventual regularity & & $\begin{array}{c}\checkmark \\
\text { (Theorem 9.2) }\end{array}$ & $\begin{array}{c}\checkmark \\
\text { (Theorem 9.2) }\end{array}$ \\
\hline
\end{tabular}

results about preservation of finiteness conditions (see also [4]). A subsemigroup $T$ of a semigroup $S$ is said to have finite Green index if it acts on its complement $S \backslash T$ in $S$ with finite quotient, in both of its natural actions via left and right multiplication (see below for a more detailed definition). The definition of Green index may also be given in terms of relative Green's relations, in the sense of Wallace [25]; see also Day [6] for a discussion of relative Green's relations in the context of the theory of topological semigroups.

Since Green index arises from the theory of relative ideals, it is natural to consider the behaviour of finiteness properties relating to ideals under taking finite Green index subsemigroups or extensions. This is our aim here. Specifically, after introducing Green index in Section 2, we consider the following finiteness conditions: stability (Section 3 ), $\mathcal{J}=\mathcal{D}$ (Section 4), having finitely many ideals (Section 5), minimal conditions (Section 6), all ideals having finite Rees index (Section 7), global torsion (Section 8) and eventual regularity (Section 9). In the process we resolve several open problems originally posed in [23] (specifically Open Problems 11.4, 11.3 (i) and 11.3 (ii)). Our main results are summarised in Tables 1 and 2. Of course, each of these results will fail to hold if the finite index assumptions are lifted.

2. Green's relations, relative relations and index. Classical Green's relations are a cornerstone of semigroup theory; their definition can be found in every semigroup monograph, such as Howie [13] or Rhodes and Steinberg [22]. They may be viewed as capturing the orbit structure with respect to the actions of a semigroup $S$ on itself 
Table 2. Summary of Rees index results

\begin{tabular}{lccc}
\hline & Finite Rees & Finite Rees \\
& $S$ arbitrary & index subsemigroups & index extensions \\
\hline $\mathcal{J}=\mathcal{D}$ & S regular & (Example 4.6) & (Theorem 4.1) \\
& & $\checkmark$ & $\checkmark$ \\
All ideals have finite Rees index & & (Theorem 4.8) & (Theorem 4.1) \\
& & $\checkmark$ & x \\
\hline
\end{tabular}

by left- and right multiplication. Relative Green's relations, introduced by Wallace [25], arise by considering the analogous orbit structure with respect to the action of a subsemigroup rather than the entire semigroup.

More specifically, let $S$ be a semigroup, and let $T$ be a subsemigroup of $S$. Denote by $S^{1}$ the semigroup obtained from $S$ by adjoining an identity element. The five relative Green's relations on $S$ with respect to $T$ are defined as follows:

$$
\begin{gathered}
u \mathcal{R}^{T} v \Leftrightarrow u T^{1}=v T^{1}, \quad u \mathcal{L}^{T} v \Leftrightarrow T^{1} u=T^{1} v, \quad u \mathcal{J}^{T} v \Leftrightarrow T^{1} u T^{1}=T^{1} v T^{1}, \\
\mathcal{H}^{T}=\mathcal{R}^{T} \cap \mathcal{L}^{T}, \quad \mathcal{D}^{T}=\mathcal{R}^{T} \circ \mathcal{L}^{T}=\mathcal{L}^{T} \circ \mathcal{R}^{T} .
\end{gathered}
$$

Each of these relations is an equivalence relation on $S$; the (relative) equivalence classes of an element $u \in S$ will be denoted by $R_{u}^{T}, L_{u}^{T}, J_{u}^{T}, H_{u}^{T}$ and $D_{u}^{T}$ respectively. Furthermore, each of these relations respects $T$, in the sense that every relative class lies wholly in $T$ or wholly in $S \backslash T$.

The following result summarises some basic facts about relative Green's relations (see [10, Proposition 4] for details).

Proposition 2.1. Let $S$ be a semigroup and let $T$ be a subsemigroup of $S$.

(i) $\mathcal{R}^{T}$ is a left congruence on $S$, and $\mathcal{L}^{T}$ is a right congruence.

(ii) For each relative $\mathcal{H}^{T}$-class $H$ either $H^{2} \cap H=\emptyset$, or $H^{2} \cap H=H$, in which case $H$ is a subgroup of $S$.

(iii) Let $u, v \in S$ be such that $u \mathcal{R}^{T} v$, and let $p, q \in T^{1}$ such that $u p=v$ and $v q=u$. Then the mapping $\rho_{p}$ given by $x \mapsto x p$ is an $\mathcal{R}^{T}$-class preserving bijection from $L_{u}^{T}$ to $L_{v}^{T}$, while the mapping $\rho_{q}$ given by $x \mapsto x q$ is an $\mathcal{R}^{T}$-class preserving bijection from $L_{v}^{T}$ to $L_{u}^{T}$, and is the inverse of the mapping $\rho_{p}$.

Following Gray and Ruškuc [10], we define the Green index of $T$ in $S$ to be one more than the number of $\mathcal{H}^{T}$-classes in $S \backslash T$. Thus, $T$ has finite Green index in $S$ if there are only finitely many $\mathcal{H}^{T}$-classes in $S \backslash T$, or, equivalently, if $S \backslash T$ contains only finitely many $\mathcal{R}^{T}$ - and $\mathcal{L}^{T}$-classes. From this it is obvious that a subsemigroup with finite Rees index must also has finite Green index. If $S$ is a group and $T$ a subgroup, the relative $\mathcal{R}^{T}$ - and $\mathcal{L}^{T}$-classes are precisely the left- and right cosets of $T$. Thus, for subgroups of groups, finite Green index coincides with the usual meaning of finite index. 
Classical Green's relations on $S$ are obtained by setting $T=S$ in the above. They and the corresponding equivalence classes are normally written without superscripts, e.g. $\mathcal{R}$ and $R_{u}$. However, since in this paper important roles will be played by both Green's equivalences and their relative versions, a peculiar notational difficulty arises. Given a semigroup $S$, a subsemigroup $T$ and $\mathcal{G} \in\{\mathcal{R}, \mathcal{L}, \mathcal{H}, \mathcal{D}, \mathcal{J}\}$, there are three versions of $\mathcal{G}$ : the 'full' relation on $S$, the 'full' relation on $T$ and the relative relation $\mathcal{G}^{T}$ on $S$. In order to resolve this formally we would need to introduce another superscript or subscript to denote the domain of the relation in question. We have adopted a slightly more informal approach: Whenever $\mathcal{G}$ appears in the text (and there is a possibility of confusion) we will always specify its domain in words (e.g. $\mathcal{G}$ on $T$, or $\mathcal{G}^{T}$ on $S$ ); the occurrences of $\mathcal{G}$ in mathematical expressions will always be accompanied by the appropriate superscript $S$ or $T$, indicating from which set the relevant multiplying elements are drawn, while the actual domain of the relation in such a situation is always possible to determine from the context.

Associated with Green's equivalences, $\mathcal{R}, \mathcal{L}$ and $\mathcal{J}$ on $S$ are three natural preorders $\leq_{\mathcal{R}}, \leq_{\mathcal{L}}$, and $\leq_{\mathcal{J}}$ on $S$ given by

$$
u \leq_{\mathcal{R}} v \text { if } u S^{1} \subseteq v S^{1}, \quad u \leq_{\mathcal{L}} v \text { if } S^{1} u \subseteq S^{1} v, \quad u \leq_{\mathcal{J}} v \text { if } S^{1} u S^{1} \subseteq S^{1} v S^{1} .
$$

These preorders induce, in the natural way, partial orders on the sets $S / \mathcal{R}, S / \mathcal{L}$ and $S / \mathcal{J}$, of $\mathcal{R}$-, $\mathcal{L}$ - and $\mathcal{J}$-classes respectively. These will all be simply denoted by $\leq$, and which one is meant will be clear from the context.

3. Stability. Stable semigroups (originally introduced in [18]) are important because they are precisely those semigroups for which the Rees-Sushkevich theorem gives a coordinatization for each $\mathcal{J}$-class. Stability is also a useful tool for proving that a semigroup satisfies the finiteness condition $\mathcal{J}=\mathcal{D}$. In particular, finite, torsion or compact Hausdorff topological semigroups are all stable. Important results regarding stability include Birget [2], and O'Carroll [21], and more recently Elston and Nehaniv [9]. For more background on stable semigroups, see [22, Appendix A.2].

We recall the following definition from [19, Proposition 3.7].

Definition 3.1. A $\mathcal{J}$-class $J$ of a semigroup $S$ is said to be right stable if it satisfies one (and hence all) of the following equivalent conditions:

(i) The set of all $\mathcal{R}$-classes in $J$ has a minimal element with respect to $\leq_{\mathcal{R}}$.

(ii) There exists $q \in J$ satisfying the following property: $q \mathcal{J} q x$ if and only if $q \mathcal{R} q x$ for all $x \in S$.

(iii) Every $q \in J$ satisfies the property stated in (ii).

(iv) Every $\mathcal{R}$-class in $J$ is minimal under $\leq_{\mathcal{R}}$ in the set of $\mathcal{R}$-classes in $J$.

We say that the whole semigroup $S$ is right stable if every $\mathcal{J}$-class of $S$ is right stable. The notion of left stability is defined dually. A $\mathcal{J}$-class and a semigroup are said to be (two-sided) stable if they are both left and right stable.

The main theorem of this section is as follows:

THEOREM 3.2. Let $S$ be a semigroup and let $T$ be a subsemigroup of $S$ with finite Green index. Then $T$ is (right, left or two-sided) stable if and only if $S$ is (right, left, or two-sided respectively) stable. 
Clearly a semigroup $S$ is left (right) stable if and only if the semigroup $S^{1}$ is left (right) stable. Hence, without loss of generality, throughout this section we will assume that $S$ has an identity 1 and that $1 \in T$.

We will need two technical lemmas.

Lemma 3.3. ([19, Proposition 3.10]) Let $S$ be a semigroup. Then $S$ is right stable if and only if $R_{a} \leq R_{b a}$ implies $R_{a}=R_{b a}$ for all $a, b \in S$.

LEMMA 3.4. Let $S$ be a semigroup, let $T$ be a right stable subsemigroup of $S$ with finite Green index, and let $a, x \in S$ such that $\left(a x^{i}, a x^{j}\right) \notin \mathcal{R}^{T}$ for all $i \neq j$. Then there exists $N \in \mathbb{N}$ such that $x^{i} \in T$ and $\left(a x^{i}, a x^{2 i}\right) \notin \mathcal{J}^{T}$ for all $i \geq N$.

Proof. Since $\left(a x^{i}, a x^{j}\right) \notin \mathcal{R}^{T}$ and $\mathcal{R}^{T}$ is a left congruence, it follows that $\left(x^{i}, x^{j}\right) \notin$ $\mathcal{R}^{T}$ for all $i \neq j$. As there are only finitely many $\mathcal{R}^{T}$-classes in $S \backslash T$, it follows that there exists $N \in \mathbb{N}$ such that $x^{i}, a x^{i} \in T$ for all $i \geq N$. Hence, the right ideal $a x^{i} T$ of $T$ properly contains the right ideal $a x^{2 i} T$ for all $i \geq N$. It follows that $R_{a x^{i}}^{T}>R_{a x^{2 i}}^{T}$. Since $T$ is right stable, and recalling Definition 3.1 (iv), $a x^{i}$ and $a x^{2 i}$ lie in distinct $\mathcal{J}$-classes of $T$. That is, $\left(a x^{i}, a x^{2 i}\right) \notin \mathcal{J}^{T}$ for all $i \geq N$.

Proof of Theorem 3.2. We prove the theorem for right stability; the proof for left stability is dual, and for the two-sided follows from these two.

$\Leftrightarrow$ Suppose that $T$ is right stable. It suffices, by Lemma 3.3, to prove that if $R_{a}^{S} \leq R_{b a}^{S}$, then $R_{a}^{S}=R_{b a}^{S}$ for all $a, b \in S$. So suppose we have $a, b, x \in S$ such that

$$
a=b a x=b^{i} a x^{i}(i \in \mathbb{N}) .
$$

We start by proving that there exist $i, j \in \mathbb{N}$ such that $i<j$ and $\left(a x^{i}, a x^{j}\right) \in \mathcal{R}^{T}$. Seeking a contradiction, assume to the contrary that $\left(a x^{i}, a x^{j}\right) \notin \mathcal{R}^{T}$ for all $i \neq j$. It follows from Lemma 3.4 that there exists $N \in \mathbb{N}$ such that $\left(a x^{i}, a x^{2 i}\right) \notin \mathcal{J}^{T}$ and $x^{i} \in T$ for all $i \geq N$. From this and

$$
a x^{i}=b^{i} \cdot a x^{2 i} \cdot 1, a x^{2 i}=1 \cdot a x^{i} \cdot x^{i},
$$

we deduce that $b^{i} \in S \backslash T$ for all $i \geq N$. Since $T$ has finite Green index, there exist $m, n \in \mathbb{N}$ such that $m-n, n \geq N$ and $\left(b^{m}, b^{n}\right) \in \mathcal{L}^{T}$, and so there exists $t \in T$ such that $b^{m}=t b^{n}$. Hence,

$$
a=b^{m} a x^{m}=t b^{n} a x^{m}=t \cdot a x^{m-n} \cdot 1, a x^{m-n}=1 \cdot a \cdot x^{m-n},
$$

and so $\left(a, a x^{m-n}\right) \in \mathcal{J}^{T}$. Similarly,

$$
a=t^{2} \cdot a x^{2(m-n)} \cdot 1, a x^{2(m-n)}=1 \cdot a \cdot x^{2(m-n)}
$$

implies that $\left(a, a x^{2(m-n)}\right) \in \mathcal{J}^{T}$. Therefore, $\left(a x^{m-n}, a x^{2(m-n)}\right) \in \mathcal{J}^{T}$, a contradiction as $m-n \geq N$.

So we have shown that there exists $i<j$ such that $\left(a x^{i}, a x^{j}\right) \in \mathcal{R}^{T}$. In particular, there exists $u \in T$ such that $a x^{i}=a x^{j} u$. It follows that

$$
b a=b^{i+1} a x^{i}=b^{i+1} a x^{j} u=a x^{j-i-1} u .
$$

Thus, from the assumption that $R_{a}^{S} \leq R_{b a}^{S}$ we obtain $(b a, a) \in \mathcal{R}^{S}$. That is, $R_{b a}^{S}=R_{a}^{S}$ as required. 
$(\Leftarrow)$ Suppose now that $S$ is right stable. We prove that $R_{a}^{T} \leq R_{b a}^{T}$ implies $R_{a}^{T}=R_{b a}^{T}$ for all $a, b \in T$. Let $a, b, x \in T$ be such that $a=b a x=b^{k} a x^{k}$. Since $R_{a}^{S} \leq R_{b a}^{S}$ and $S$ is right stable, it follows that $R_{a}^{S}=R_{b a}^{S}$. Hence, there exists $y \in S$ such that $b a=a y$ (and so $b^{k} a=a y^{k}$ for all $k \geq 1$ ). Now

$$
b a=b^{k+1} a x^{k}=a y^{k+1} x^{k} .
$$

If $y^{k+1} x^{k} \in T$ for some $k \geq 1$, then $b a \in a T$ by (1). Hence, $R_{a}^{T}=R_{b a}^{T}$ and the proof is complete.

On the other hand, suppose that $y^{k+1} x^{k} \in S \backslash T$ for all $k \geq 1$. Then $y^{k} \in S \backslash T$ for all $k \geq 2($ as $x \in T)$. Then, since $T$ has finite Green index, there exist $m \geq 2$ and $n \geq 1$ such that $\left(y^{m+n}, y^{m}\right) \in \mathcal{L}^{T}$. Hence, there exists $t \in T$ such that $y^{m+n}=t y^{m}$. Then for all $k \geq 1$ we have

$$
t^{k} y^{m} x^{m+k n-1}=y^{m+k n} x^{m+k n-1} \in S \backslash T .
$$

It follows that $y^{m} x^{m+k n-1} \in S \backslash T$ for all $k \geq 1$ (as $t \in T$ ). Hence, again since $T$ has finite Green index, there exist $u, v \in \mathbb{N}$ such that $v>u+1$ and $\left(y^{m} x^{m+u n-1}, y^{m} x^{m+v n-1}\right) \in$ $\mathcal{R}^{T}$, and so there exists $t_{0} \in T$ where

$$
y^{m} x^{m+u n-1}=y^{m} x^{m+v n-1} t_{0} .
$$

To conclude, we have

$$
\begin{aligned}
b a & =a y^{m+u n} x^{m+u n-1}=a t^{u} y^{m} x^{m+u n-1}=a t^{u} y^{m} x^{m+v n-1} t_{0} \\
& =a t^{u} y^{m} x^{m+u n-1} \cdot x^{(v-u) n} t_{0}=b a \cdot x^{(v-u) n} t_{0}=b a x \cdot x^{(v-u) n-1} t_{0} \\
& =a x^{(v-u) n-1} t_{0} \in a T,
\end{aligned}
$$

where (1), (2) and (3) have been used in the first three steps above. Thus, $R_{a}^{T}=R_{b a}^{T}$ as required.

4. The property $\mathcal{J}=\mathcal{D}$. Many natural classes of semigroups have the property that the relations $\mathcal{J}$ and $\mathcal{D}$ coincide. For instance, this is the case for the full transformation monoid of all maps from a set to itself, for the monoid of all linear transformations on a vector space and also every stable (and in particular every finite) semigroup.

Given a semigroup $S$ and subsemigroup $T$ of finite Rees index, it was asked in Ruškuc [23, Open Problem 11.4] whether it is true that the relations $\mathcal{J}$ and $\mathcal{D}$ coincide in $S$ if and only if they coincide in $T$. In this section we will show that this problem has a positive solution in one direction, when passing from $T$ to $S$, even under the weaker assumption of finite Green index. On the other hand, rather surprisingly, we will see that the converse does not hold, by exhibiting a semigroup $S$ and subsemigroup $T$ such that $|S \backslash T|=1$, where the relations $\mathcal{J}$ and $\mathcal{D}$ coincide in $S$ but do not coincide in $T$. However, we will see that by placing regularity assumptions on $S$ or $T$, respectively, positive results in this direction may be recovered.

We begin by establishing the following.

THEOREM 4.1. Let $S$ be a semigroup, and let $T$ be a subsemigroup of $S$ with finite Green index. If $\mathcal{J}=\mathcal{D}$ in $T$, then $\mathcal{J}=\mathcal{D}$ in $S$. 
In order to prove Theorem 4.1 we need some preparation. Let $S$ be a semigroup and $T$ be a subsemigroup of $S$ with finite Green index such that $\mathcal{J}=\mathcal{D}$ in $T$. Note that $\mathcal{J}=\mathcal{D}$ in $S$ if and only if $\mathcal{J}=\mathcal{D}$ in $S^{1}$. Hence, as in the previous section, throughout this section we assume without loss of generality that $S$ has an identity 1 and that $1 \in T$. For any pair $a, b \in S$ with $(a, b) \in \mathcal{J}^{S}$ define

$$
Q_{a, b}=\left\{\left(x_{1}, x_{2}, y_{1}, y_{2}\right) \in S \times S \times S \times S: a=x_{1} b y_{1} \text { and } b=x_{2} a y_{2}\right\} .
$$

Note that

$$
\begin{aligned}
& \left(x_{1}, x_{2}, y_{1}, y_{2}\right) \in Q_{a, b} \Rightarrow \\
& \left(x_{1}\left(x_{2} x_{1}\right)^{k}, x_{2}, y_{1}\left(y_{2} y_{1}\right)^{k}, y_{2}\right),\left(x_{1}, x_{2}\left(x_{1} x_{2}\right)^{k}, y_{1}, y_{2}\left(y_{1} y_{2}\right)^{k}\right) \in Q_{a, b} .
\end{aligned}
$$

LemmA 4.2. Let $a, b \in S$ such that $(a, b) \in \mathcal{J}^{S}$ and let $\left(x_{1}, x_{2}, y_{1}, y_{2}\right) \in Q_{a, b}$. Then:

(i) if the set

$$
\left\{k \in \mathbb{N}: x_{1}\left(x_{2} x_{1}\right)^{k} \in S \backslash T \text { or } x_{2}\left(x_{1} x_{2}\right)^{k} \in S \backslash T\right\}
$$

is infinite, then $\left(b, x_{1} b\right),\left(a, x_{2} a\right) \in \mathcal{L}^{S}$;

(ii) if the set

$$
\left\{k \in \mathbb{N}: y_{1}\left(y_{2} y_{1}\right)^{k} \in S \backslash T \text { or } y_{2}\left(y_{1} y_{2}\right)^{k} \in S \backslash T\right\}
$$

is infinite, then $\left(b, b y_{1}\right),\left(a, a y_{2}\right) \in \mathcal{R}^{S}$.

Proof. It suffices to prove (i) assuming $x_{1}\left(x_{2} x_{1}\right)^{k} \in S \backslash T$ for infinitely many $k$. Because $T$ has finite Green index in $S$, we have

$$
\left(x_{1}\left(x_{2} x_{1}\right)^{k}, x_{1}\left(x_{2} x_{1}\right)^{k+r}\right) \in \mathcal{L}^{T}
$$

for some $k, r>0$, and so there exists $t \in T$ such that $x_{1}\left(x_{2} x_{1}\right)^{k}=t x_{1}\left(x_{2} x_{1}\right)^{k+r}$. Hence,

$$
b=\left(x_{2} x_{1}\right)^{k+1} b\left(y_{1} y_{2}\right)^{k+1}=x_{2} t x_{1}\left(x_{2} x_{1}\right)^{k+r} b\left(y_{1} y_{2}\right)^{k+1}=x_{2} t x_{1}\left(x_{2} x_{1}\right)^{r-1} b
$$

and so $\left(b, x_{1} b\right) \in \mathcal{L}^{S}$. Also,

$$
a=\left(x_{1} x_{2}\right)^{k+1} a\left(y_{2} y_{1}\right)^{k+1}=t x_{1}\left(x_{2} x_{1}\right)^{k+r} x_{2} a\left(y_{2} y_{1}\right)^{k+1}=t\left(x_{1} x_{2}\right)^{r} a
$$

and so $\left(a, x_{2} a\right) \in \mathcal{L}^{S}$.

Lemma 4.3. Let $a, b \in S$ be such that $(a, b) \in \mathcal{J}^{S}$. If there exists $\left(x_{1}, x_{2}, y_{1}, y_{2}\right) \in$ $Q_{a, b}$ with $x_{1}, x_{2}, y_{1}, y_{2} \in T$, then $(a, b) \in \mathcal{D}^{S}$.

Proof. From $\left(x_{1}, x_{2}, y_{1}, y_{2}\right) \in Q_{a, b}$ and $x_{1}, x_{2}, y_{1}, y_{2} \in T$, it follows that $a \in T$ if and only if $b \in T$. If $a, b \in T$, we have $(a, b) \in \mathcal{J}^{T}$ and so $(a, b) \in \mathcal{D}^{T}$ by assumption. Thus, $(a, b) \in \mathcal{D}^{S}$ as required.

Consider now the case where $a, b \in S \backslash T$. It will suffice to prove that $\left(b, x_{1} b\right) \in \mathcal{L}^{S}$ and $\left(x_{1} b, a\right) \in \mathcal{R}^{S}$. Since $b=\left(x_{2} x_{1}\right)^{k} b\left(y_{1} y_{2}\right)^{k}, b \notin T$ and $y_{1}, y_{2} \in T$, we have $\left(x_{2} x_{1}\right)^{k} b \in$ $S \backslash T$ for all $k \geq 1$. Finite Green index implies that there exist $m, n \geq 1$ and $t \in T$ with $\left(x_{2} x_{1}\right)^{m} b=t\left(x_{2} x_{1}\right)^{m+n} b$. Then

$$
b=\left(x_{2} x_{1}\right)^{m} b\left(y_{1} y_{2}\right)^{m}=t\left(x_{2} x_{1}\right)^{m+n} b\left(y_{1} y_{2}\right)^{m}=t\left(x_{2} x_{1}\right)^{n} b
$$


and so $\left(b, x_{1} b\right) \in \mathcal{L}^{S}$. Analogously, $\left(b, b y_{1}\right) \in \mathcal{R}^{S}$ and since $\mathcal{R}^{S}$ is a left congruence,

$$
\left(a, x_{1} b\right)=\left(x_{1} b y_{1}, x_{1} b\right) \in \mathcal{R}^{S},
$$

completing the proof.

LeMmA 4.4. Let $a, b \in S$ such that $(a, b) \in \mathcal{J}^{S}$. If $\left(x_{1}, 1, y_{1}, y_{2}\right) \in Q_{a, b}$ with $y_{1}, y_{2} \in$ $T$, then $(a, b) \in \mathcal{D}^{S}$.

Proof. As $\left(x_{1}, 1, y_{1}, y_{2}\right) \in Q_{a, b}$, we have $a=x_{1} b y_{1}$ and $b=a y_{2}$. By (4) we have $\left(x_{1}^{k}, 1, y_{1}\left(y_{2} y_{1}\right)^{k-1}, y_{2}\right) \in Q_{a, b}$ for all $k \geq 1$. Hence, if there exists $k \in \mathbb{N}$ such that $x_{1}^{k} \in T$, then $(a, b) \in \mathcal{D}^{S}$ by Lemma 4.3.

Thus, we may assume that $x_{1}^{k} \in S \backslash T$ for all $k \geq 1$. Finite Green index of $T$ in $S$ implies that there exists $m, n \geq 1$ such that $x_{1}^{m+n}=t x_{1}^{m}$ for some $t \in T$. Hence, since $x_{1}^{m} b\left(y_{1} y_{2}\right)^{m}=b$, we have

$$
a=x_{1}^{m+n} b\left(y_{1} y_{2}\right)^{m+n-1} y_{1}=t x_{1}^{m} b\left(y_{1} y_{2}\right)^{m+n-1} y_{1}=t \cdot b \cdot\left(y_{1} y_{2}\right)^{n-1} y_{1} .
$$

It follows that $\left(t, 1,\left(y_{1} y_{2}\right)^{n-1} y_{1}, y_{2}\right) \in Q_{a, b}$, and, since all the entries are in $T$, the result follows from Lemma 4.3.

The following lemma provides the crucial step in the proof of Theorem 4.1.

Lemma 4.5. Let $a, b \in S$ such that $(a, b) \in \mathcal{J}^{S}$. If $\left(x_{1}, x_{2}, y_{1}, y_{2}\right) \in Q_{a, b}$ with $y_{1}, y_{2} \in$ $T$, then $(a, b) \in \mathcal{D}^{S}$.

Proof. There are two cases to consider:

(1) There exists $N \in \mathbb{N}$ such that $x_{1}\left(x_{2} x_{1}\right)^{k}, x_{2}\left(x_{1} x_{2}\right)^{k} \in T$ for all $k \geq N$; and

(2) $x_{1}\left(x_{2} x_{1}\right)^{k} \in S \backslash T$ or $x_{2}\left(x_{1} x_{2}\right)^{k} \in S \backslash T$ for infinitely many $k$.

In case (1), the quadruple

$$
\left(x_{1}\left(x_{2} x_{1}\right)^{N}, x_{2}\left(x_{1} x_{2}\right)^{N}, y_{1}\left(y_{2} y_{1}\right)^{N}, y_{2}\left(y_{1} y_{2}\right)^{N}\right)
$$

lies in $Q_{a, b}$ and all of its entries are in $T$. Hence, the result follows from Lemma 4.3.

To prove the lemma in case (2), note that $x_{2} a=x_{2} x_{1} \cdot b \cdot y_{1}$ and $b=1 \cdot x_{2} a \cdot y_{2}$. This implies that $\left(x_{2} a, b\right) \in \mathcal{J}^{S}$ and $\left(x_{2} x_{1}, 1, y_{1}, y_{2}\right) \in Q_{x_{2} a, b}$. So by Lemma 4.4 $\left(x_{2} a, b\right) \in \mathcal{D}^{S}$. By the assumption of case (2) it follows from Lemma 4.2 (i) that $\left(x_{2} a, a\right) \in \mathcal{L}^{S}$. Therefore, $(a, b) \in \mathcal{D}^{S}$.

We can now use Lemmas 4.2 and 4.5 to prove Theorem 4.1.

Proof of Theorem 4.1 Let $a, b \in S$ such that $(a, b) \in \mathcal{J}^{S}$. Then by Lemma 4.5 (and its dual), if there exists $\left(x_{1}, x_{2}, y_{1}, y_{2}\right) \in Q_{a, b}$ with either $x_{1}, x_{2} \in T$ or $y_{1}, y_{2} \in T$, then the proof is complete.

If neither of these conditions hold, then for all $\left(x_{1}, x_{2}, y_{1}, y_{2}\right) \in Q_{a, b}$ and for all $k \in \mathbb{N}$ we have

$$
\left(x_{1}\left(x_{2} x_{1}\right)^{k} \in S \backslash T \text { or } x_{2}\left(x_{1} x_{2}\right)^{k} \in S \backslash T\right) \text { and }\left(y_{1}\left(y_{2} y_{1}\right)^{k} \in S \backslash T \text { or } y_{2}\left(y_{1} y_{2}\right)^{k} \in S \backslash T\right)
$$

by (4). Therefore, by Lemma 4.2, $\left(b, x_{1} b\right) \in \mathcal{L}^{S}$ and $\left(b, b y_{1}\right) \in \mathcal{R}^{S}$. Thus, $\left(a, b y_{1}\right)=$ $\left(x_{1} b \cdot y_{1}, b \cdot y_{1}\right) \in \mathcal{L}^{S}$ and so $(a, b) \in \mathcal{D}^{S}$. 
The property $\mathcal{J}=\mathcal{D}$ is not inherited the other way round, from $S$ to $T$, even when $T$ has finite Rees index as the following example shows.

EXAMPLE 4.6. We are going to define a semigroup $S$ by means of a (fairly large) presentation. The generators are

$$
A=\{a, b, c, d, x\}
$$

and the main relations are

$$
b x a=a c, a c d=a, d c=c d, x^{3}=x, x^{2} a=a .
$$

There are also a number of zero relations, making the 'unnecessary' products of generators equal to zero:

$$
\begin{aligned}
& a a=a b=a x=0, \\
& b a=b b=b c=b d=0, \\
& c a=c b=c x=0, \\
& d a=d b=d x=0, \\
& x c=x d=0, \\
& b x^{2} b=0 .
\end{aligned}
$$

Note also that

$$
a c^{k+1} d=a c d c^{k}=a c^{k}
$$

for all $k \geq 0$. A routine check confirms that the presentation together with the relations $a c^{k+1} d=a c^{k}$, viewed as a string rewriting system, is confluent. (See [3] for definitions relating to rewriting systems.) It is easy to see that this rewriting system is also terminating: indeed, it is length reducing, except for the relation $d c=c d$, which pushes $d$ s systematically to the right. Therefore, a set of normal forms is provided by all the words from $A^{+}$which do not contain the left-hand side of a relation as a subword; they are:

$$
\begin{aligned}
& x, x^{2} \\
& a c^{i}, a d^{j}, x a c^{i}, x a d^{j}, c^{i} d^{j}(i, j \geq 0), \\
& x^{j}(b x)^{i} b^{k} x^{l}(i \geq 0, j=0,1,2, k=0,1, l=0,1,2) .
\end{aligned}
$$

(with the empty word excluded).

Computing the non-singleton Green's classes in $S$ we obtain:

$$
\begin{aligned}
& L_{x}^{S}=R_{x}^{S}=D_{x}^{S}=J_{x}^{S}=\left\{x, x^{2}\right\}, \\
& L_{a c^{i}}^{S}=\left\{a c^{i}, x a c^{i}\right\}, L_{a d^{j}}^{S}=\left\{a d^{j}, x a d^{j}\right\}, \\
& R_{a}^{S}=\left\{a c^{i}, a d^{i}: i \geq 0\right\}, R_{x a}^{S}=\left\{x a c^{i}, x a d^{i}: i \geq 0\right\}, \\
& J_{a}^{S}=D_{a}^{S}=\left\{a c^{i}, a d^{i}, x a c^{i}, x a d^{i}: i \geq 0\right\} .
\end{aligned}
$$

The remaining non-singleton Green's classes in $S$ arise from the remaining normal form words that begin or end in $x$, that is, those of the form $x \alpha x, x \alpha b$ or $b \alpha x$, where 
Table 3. The action of generators on the elements of $S$ via right multiplication in Example 4.6

\begin{tabular}{|c|c|c|c|c|c|}
\hline & $\mathrm{a}$ & $\mathrm{b}$ & $\mathrm{c}$ & $\mathrm{d}$ & $\mathrm{x}$ \\
\hline $\mathrm{x}$ & xa & $\mathrm{xb}$ & 0 & 0 & $x^{2}$ \\
\hline$x^{2}$ & $\mathrm{a}$ & $x^{2} b$ & 0 & 0 & $\mathrm{x}$ \\
\hline $\mathrm{a}$ & 0 & 0 & $\mathrm{ac}$ & $\mathrm{ad}$ & 0 \\
\hline$a c^{i}$ & 0 & 0 & $a c^{i+1}$ & $a c^{i-1}$ & 0 \\
\hline$a d^{j}$ & 0 & 0 & $a d^{i-1}$ & $a d^{i+1}$ & 0 \\
\hline$c^{i} d^{j}$ & 0 & 0 & $c^{i+1} d^{j}$ & $c^{i} d^{j+1}$ & 0 \\
\hline xa & 0 & 0 & xac & xad & 0 \\
\hline$x a c^{i}$ & 0 & 0 & $x a c^{i+1}$ & $x a c^{i-1}$ & 0 \\
\hline$x a d^{j}$ & 0 & 0 & $x a d^{i-1}$ & $x a d^{i+1}$ & 0 \\
\hline$x^{j}(b x)^{i} b^{k} x^{l}$ & ee below & see below & 0 & 0 & $x^{j}(b x)^{i} b^{k} x^{l+1}$ \\
\hline$x^{j}(b x)^{i} b^{k} x^{l} \cdot a=$ & $=\left\{\begin{array}{l}a c^{i} \\
x a c^{i} \\
0\end{array}\right.$ & $\begin{array}{l}\text { if } k=l, j=0,2 \\
\text { if } k=l, j=1 \\
\text { otherwise }\end{array}$ & $x^{j}(b x)^{i} b$ & $b=$ & $\begin{array}{l}x^{l} b, \quad \text { if } k=l \\
\text { otherwise }\end{array}$ \\
\hline
\end{tabular}

$\alpha \in A^{*}$. These elements give rise to the following non-trivial Green's classes in $S$

$$
\begin{aligned}
& R_{x \alpha x}^{S}=\left\{x \alpha x, x \alpha x^{2}\right\}, L_{x \alpha x}^{S}=\left\{x \alpha x, x^{2} \alpha x\right\}, \\
& D_{x \alpha x}^{S}=J_{x \alpha x}^{S}=\left\{x \alpha x, x \alpha x^{2}, x^{2} \alpha x, x^{2} \alpha x^{2}\right\}, \\
& L_{x \alpha b}^{S}=D_{x \alpha b}^{S}=J_{x \alpha b}^{S}=\left\{x \alpha b, x^{2} \alpha b\right\}, \\
& R_{b \alpha x}^{S}=D_{b \alpha x}^{S}=J_{b \alpha x}^{S}=\left\{b \alpha x, b \alpha x^{2}\right\} .
\end{aligned}
$$

Following are two useful observations that can be used for the verification of these claims:

- If $u$ and $w$ are non-zero words both representing non-zero elements of $S$, and $u=w$ in $S$, then $u$ and $w$ must contain the same number of occurrences of the letter $a$.

- If $u$ and $w$ are words both representing the same element of $S$, then $u$ contains a letter different from $x$ if and only if $w$ contains a letter different from $x$.

The above claims about Green's classes $\mathcal{R}, \mathcal{L}$ and $\mathcal{D}$ in $S$ can all now be easily read off from Tables 3 and 4 . Of the remaining claims, the most important is that $J_{a}^{S}=D_{a}^{S}$, so let us now see why this is so. Clearly $D_{a}^{S} \subseteq J_{a}^{S}$. For the converse, suppose that $w \mathcal{J}^{S} a$, where $w$ is a normal form word. This means that there are normal form words $\alpha, \beta, \gamma, \delta$ such that

$$
\alpha w \beta=a \text { and } \gamma a \delta=w
$$

in $S$. From $\alpha w \beta=a$ it follows that the word $\alpha w \beta$ contains exactly one occurrence of the letter $a$. But $\gamma a \delta=w$ tells us that $w$ contains at least one occurrence of the letter $a$. Therefore, $w$ must contain exactly one occurrence of the letter $a$, and thus looking 
Table 4. The action of generators on the elements of $S$ via left multiplication in Example 4.6

\begin{tabular}{|c|c|c|c|c|c|c|c|c|c|c|}
\hline & $\mathrm{x}$ & $x^{2}$ & $\mathrm{a}$ & $a c^{i}$ & $a d^{j}$ & $c^{i} d^{j}$ & xa & $x a c^{i}$ & $x a d^{j}$ & $x^{j}(b x)^{i} b^{k} x^{l}$ \\
\hline $\mathrm{a}$ & 0 & 0 & 0 & 0 & 0 & $a c^{i-1} d^{i-1}$ & 0 & 0 & 0 & 0 \\
\hline$b$ & $\mathrm{bx}$ & $b x^{2}$ & 0 & 0 & 0 & 0 & $\mathrm{ac}$ & $a c^{i+1}$ & $a d^{j-1}$ & see below \\
\hline $\mathrm{c}$ & 0 & 0 & 0 & 0 & 0 & $c^{i+1} d^{j}$ & 0 & 0 & 0 & 0 \\
\hline d & 0 & 0 & 0 & 0 & 0 & $c^{i} d^{j+1}$ & 0 & 0 & 0 & 0 \\
\hline \multirow[t]{2}{*}{$x$} & $x^{2}$ & $\mathrm{x}$ & xa & $x a c^{i}$ & $x a d^{j}$ & 0 & $\mathrm{a}$ & $a c^{i}$ & $a d^{j}$ & $x^{j+1}(b x)^{i} b^{k} x^{l}$ \\
\hline & & & \multicolumn{7}{|c|}{$\begin{array}{ll}b x^{j}(b x)^{i} b^{k} x^{l}, & \text { if } j=1 \text { or } j=i=k=0 \\
0, & \text { otherwise }\end{array}$} & \\
\hline
\end{tabular}

at the list of normal form words we conclude that $w$ belongs to the set

$$
\left\{a c^{i}, a d^{i}, x a c^{i}, x a d^{i}: i \geq 0\right\}=D_{a}^{S} .
$$

Therefore, $J_{a}^{S}=D_{a}^{S}$. The claims about the remaining non-trivial $\mathcal{D}$ - and $\mathcal{J}$-classes are easily verified, and we conclude $\mathcal{J}^{S}=\mathcal{D}^{S}$.

Let now $T=S \backslash\{x\}$. The only words of $S$ that are equal to $x$ are $x^{2 i+1}$, where $i \geq 1$. Such a word cannot be expressed as a product of two elements of $T$. Hence, $T$ is a subsemigroup of $S$. Now note that

$$
a=b \cdot x a \cdot d, x a=x b x \cdot a \cdot d ;
$$

hence, $(a, x a) \in \mathcal{J}^{T}$. We claim that $(a, x a) \notin \mathcal{D}^{T}$. As in $S$ we have

$$
R_{a}^{T}=\left\{a c^{i}, a d^{i}: i \geq 0\right\} .
$$

However, unlike the situation in $S$, the $\mathcal{L}$-class of $x a$ in $T$ is trivial. Indeed, looking at Table 4 we see that the only elements of $T$ we can premultiply $x a$ with and not obtain 0 are of the form $b,(x b)^{i},(x b)^{i} x^{2}, x(x b)^{i}, x(x b)^{i} x^{2}$. After rewriting we obtain the words $a c^{i}$ and $x a c^{i}$, where $i \geq 1$. Thus, by premultiplying $x a$ by elements of $T$ we never get back to $x a$, and so $L_{x a}^{T}$ is trivial. Therefore, $L_{x a}^{T} \cap R_{a}^{T}=\emptyset$, and hence $(a, x a) \notin \mathcal{D}^{T}$.

The situation is made even more curious by the fact that the property $\mathcal{J}=\mathcal{D}$ is inherited by subsemigroups of finite Green index if certain regularity assumptions are made on $S$ or $T$. Below are two sample results. We have not been able to obtain a unified general result.

THEOREM 4.7. Let $T$ be a regular subsemigroup of finite Green index in a semigroup $S$. Then $\mathcal{J}=\mathcal{D}$ in $S$ implies $\mathcal{J}=\mathcal{D}$ in $T$.

THEOREM 4.8. Let $T$ be a subsemigroup of finite Rees index in a regular semigroup $S$. Then $\mathcal{J}=\mathcal{D}$ in $S$ implies $\mathcal{J}=\mathcal{D}$ in $T$.

In order to prove Theorems 4.7 and 4.8 we need the following technical lemma:

LEMMA 4.9. Let $S$ be a semigroup, let $T$ be a subsemigroup of finite Green index in $S$, and let $a, b \in T$ be such that $a \mathcal{D}^{S}$ ab. Then there exists $d \in T$ such that $a \mathcal{L}^{S} d \mathcal{R}^{S} a b$ and $d \mathcal{J}^{T} a$. 
Proof. Since $a \mathcal{D}^{S} a b$, there exists $c \in S$ such that $a \mathcal{L}^{S} c \mathcal{R}^{S} a b$. This means that there exist $x_{1}, x_{2}, y_{1}, y_{2} \in S$ with

$$
a=x_{1} c, c=y_{1} a, a b=c x_{2}, c=a b y_{2} .
$$

Then $a=x_{1} \cdot a \cdot b y_{2}$ and $c=x_{1} \cdot c \cdot b y_{2}$.

There are two cases to consider.

Case 1. There are infinitely many $k \geq 1$ with $\left(b y_{2}\right)^{k} \in S \backslash T$. Then, since $T$ has finite Green index in $S$, there exist $k, n \geq 1$ such that $\left(b y_{2}\right)^{k} \mathcal{R}^{T}\left(b y_{2}\right)^{k+n}$. In particular, there exists $t \in T$ such that $\left(b y_{2}\right)^{k+n} t=\left(b y_{2}\right)^{k}$. Then

$$
a=x_{1}^{k} a\left(b y_{2}\right)^{k}=x_{1}^{k} a\left(b y_{2}\right)^{k+n} t=a\left(b y_{2}\right)^{n} t=c \cdot\left(b y_{2}\right)^{n-1} t .
$$

Together with $c=a \cdot b y_{2}$, we obtain $a \mathcal{R}^{S} c$. Then $a \mathcal{R}^{S} a b$ and the assertion holds with $d=a$.

Case 2. There exists $k_{0} \geq 1$ such that $\left(b y_{2}\right)^{k} \in T$ for all $k \geq k_{0}$. We claim that the assertion of the lemma holds for $d=c$. We prove first that $c \in T$. Suppose the converse: $c \in S \backslash T$, and recall that $c=x_{1}^{k} c \cdot\left(b y_{2}\right)^{k}$. Hence, $x_{1}^{k} c \in S \backslash T$ for all $k \geq k_{0}$. Then there exist $k, n \geq k_{0}$ such that $x_{1}^{k} c \mathcal{L}^{T} x_{1}^{k+n} c$. In particular, $t x_{1}^{k} c=x_{1}^{k+n} c$ for some $t \in T$. Then

$$
c=x_{1}^{k+n} c\left(b y_{2}\right)^{k+n}=t x_{1}^{k} c\left(b y_{2}\right)^{k+n}=t x_{1} \cdot c \cdot\left(b y_{2}\right)^{n+1}=t \cdot a \cdot\left(b y_{2}\right)^{n+1} \text {. }
$$

Since $n \geq k_{0}$, we obtain $c \in T$, a contradiction. Hence, $c \in T$. It remains to prove that $c \mathcal{J}^{T} a$.

Now we have $c=x_{1}^{k} \cdot a \cdot\left(b y_{2}\right)^{k+1}$ for all $k \geq k_{0}$. If there are infinitely many $k$ such that $x_{1}^{k} \in S \backslash T$ then there exist $k, n \geq k_{0}$ such that $x_{1}^{k+n}=t \cdot x_{1}^{k}$ for some $t \in T$. Then

$$
c=x_{1}^{k+n} \cdot a \cdot\left(b y_{2}\right)^{k+n+1}=t x_{1}^{k} \cdot a \cdot\left(b y_{2}\right)^{k+n+1}=t \cdot a \cdot\left(b y_{2}\right)^{n+1}
$$

and so $c \in T a T$. On the other hand, if $x_{1}^{k} \in T$ for all $k \geq N_{0}$ for some $N_{0} \geq k_{0}$, then $c=x_{1}^{N_{0}} \cdot a \cdot\left(b y_{2}\right)^{N_{0}+1}$ and so $c \in T a T$.

Having $a=x_{1}^{k+1} \cdot c \cdot\left(b y_{2}\right)^{k}$ for all $k \geq k_{0}$, by analogous reasoning as in the previous paragraph we deduce that $a \in T c T$. Thus, $c \mathcal{J}^{T} a$ as required.

Proof of Theorem 4.7. Suppose that $\mathcal{J}=\mathcal{D}$ in $S$. Take two $\mathcal{J}^{T}$-equivalent elements $t_{1}$ and $t_{2}$ of $T$. Then there exist elements $\alpha_{1}, \alpha_{2}, \beta_{1}, \beta_{2} \in T$ with $t_{1}=\alpha_{1} t_{2} \beta_{1}$ and $t_{2}=$ $\alpha_{2} t_{1} \beta_{2}$. Then $t_{1} \mathcal{J}^{T} t_{1} \beta_{2} \mathcal{J}^{T} t_{2}$ and $t_{2}=\alpha_{2} \cdot t_{1} \beta_{2}$. Hence, to prove the theorem, it suffices to establish that, for every two elements $a, b \in T$, if $a \mathcal{J}^{T} a b$ then $a \mathcal{D}^{T} a b$, and that if $a \mathcal{J}^{T} b a$ then $a \mathcal{D}^{T} b a$. We will only prove the first assertion, the second follows by a similar argument.

So suppose that $a, b \in T$ are such that $a \mathcal{J}^{T} a b$. Then $a \mathcal{J}^{S} a b$ and so $a \mathcal{D}^{S} a b$. By Lemma 4.9 we have $a \mathcal{L}^{S} c \mathcal{R}^{S} a b$ for some $c \in T$. Now, since $T$ is regular, we have $a \mathcal{L}^{T} c$ and $c \mathcal{R}^{T} a b$ (see [22, Proposition A.1.16]). Thus, $a \mathcal{D}^{T} a b$ and we are done.

Before proving Theorem 4.8 we need another technical result.

Lemma 4.10. Let $T$ be a semigroup and $x, y, \alpha, \beta, \gamma, \delta \in T$ such that $x=\alpha y \beta$ and $y=\gamma x \delta$. Furthermore, assume that there exists $n \in \mathbb{N}$ such that $y=y(\beta \delta)^{n}$. Then $x$ and $y$ are $\mathcal{D}$-related in $T$. 
Proof. First notice that $y \mathcal{R}^{T} y \beta$. Now

$$
y=(\gamma \alpha)^{n} \cdot y \cdot(\beta \delta)^{n}=(\gamma \alpha)^{n} y .
$$

Hence, $y \beta=(\gamma \alpha)^{n-1} \gamma \cdot \alpha y \beta=(\gamma \alpha)^{n-1} \gamma \cdot x$. Since $x=\alpha \cdot y \beta$, we obtain $y \beta \mathcal{L}^{T} x$. Thus, $x \mathcal{D}^{T} y$.

Proof of Theorem 4.8. Suppose that $\mathcal{J}=\mathcal{D}$ in $S$. As in the proof of Theorem 4.7, it suffices to prove that if $a \mathcal{J}^{T} a b$ then $a \mathcal{D}^{T} a b$ for all $a, b \in T$. So let $a \mathcal{J}^{T} a b$ for some $a, b \in T$. Then $a \mathcal{D}^{S} a b$. By Lemma 4.9 we have that there exists $c \in J_{a}^{T}=J_{a b}^{T}$ such that $a \mathcal{L}^{S} c \mathcal{R}^{S} a b$.

Therefore, in order to prove the theorem, it is enough to prove that if $x, y \in T$ are such that $x \mathcal{R}^{S} y$ (or $x \mathcal{L}^{S} y$ ) and $x \mathcal{J}^{T} y$, then $x \mathcal{D}^{T} y$. We will do this only in the case of $\mathcal{R}$, the other case follows by symmetry.

So let $x, y \in T$ be such that $x \mathcal{J}^{T} y$ and $x \mathcal{R}^{S} y$. Since $S$ is regular, there exist $x^{\prime}, y^{\prime} \in S$ such that $x=x x^{\prime} x$ and $y=y y^{\prime} y$. There also exist $\alpha, \beta, \gamma, \delta \in T$ such that $x=\alpha y \beta$ and $y=\gamma x \delta$. There are four cases.

Case 1. $x \in y T$ and $y \in x T$. Then immediately $x \mathcal{R}^{T} y$ as required.

Case 2. $x=y t$ and $y=x f$ for some $t \in T$ and $f \in S \backslash T$. In this case we distinguish the following three subcases:

Subcase 2a. $x^{\prime} \in T$ and $y^{\prime} \in T$. Then $x \mathcal{R}^{T} x x^{\prime}$ and $y \mathcal{R}^{T} y y^{\prime}$. In addition, $x x^{\prime} \mathcal{R}^{S} x \mathcal{R}^{S} y \mathcal{R}^{S} y y^{\prime}$. Hence, since an idempotent is a left identity in its $\mathcal{R}$-class, $x x^{\prime}=y y^{\prime} \cdot x x^{\prime}$ and $y y^{\prime}=x x^{\prime} \cdot y y^{\prime}$. Therefore, $x x^{\prime} \mathcal{R}^{T} y y^{\prime}$ and so $x \mathcal{R}^{T} y$.

Subcase 2b. $x^{\prime} \in S \backslash T$ and $y^{\prime} \in T$. Then $y \mathcal{R}^{T} y y^{\prime}$. Moreover,

$$
\begin{aligned}
x & =y y^{\prime} \cdot y t, \\
y y^{\prime} & =x \cdot f y^{\prime} .
\end{aligned}
$$

If $f y^{\prime} \in T$ then $x \mathcal{R}^{T} y y^{\prime}$ and so $x \mathcal{R}^{T} y$. So, suppose $f y^{\prime} \in S \backslash T$. Recall that $x \mathcal{R}^{S} y y^{\prime}$ and $x \mathcal{J}^{T} y y^{\prime}$. Since it suffices to prove that $x \mathcal{D}^{T} y y^{\prime}$ and since $y y^{\prime}$ is an idempotent, in view of (6) we may assume that $y^{2}=y=y^{\prime}$. Now (7) becomes $y=x \cdot f y$. If $f y \in T$ then $x \mathcal{R}^{T} y$ as required. Hence, we may assume that $f y \in S \backslash T$. Then $f(\gamma \alpha)^{k} \cdot y(\beta \delta)^{k} \in S \backslash T$ for all $k \geq 1$ and so, since $\beta, \delta \in T$, it follows that $f(\gamma \alpha)^{k} \in S \backslash T$ for all $k \geq 1$. Since $T$ has finite Rees index in $S$, this implies that $f(\gamma \alpha)^{k}=f(\gamma \alpha)^{k+n}$ for some $k, n \geq 1$. Then

$$
f y=f \cdot(\gamma \alpha)^{k+n} y(\beta \delta)^{k+n}=f(\gamma \alpha)^{k} y(\beta \delta)^{k+n}=f y(\beta \delta)^{n} .
$$

Hence, $y=x \cdot f y=x f y(\beta \delta)^{n}=y(\beta \delta)^{n}$ and so by Lemma $4.10, x \mathcal{D}^{T} y$ as required.

Subcase 2c. $y^{\prime} \in S \backslash T$. Then $y=x \cdot f y^{\prime} y$. If $f y^{\prime} y \in T$, then $x \mathcal{R}^{T} y$. Hence, we may assume that $f y^{\prime} y \in S \backslash T$. Then $f y^{\prime}(\gamma \alpha)^{k} \cdot y(\beta \gamma)^{k} \in S \backslash T$ for all $k \geq 1$. Thus, $f y^{\prime}(\gamma \alpha)^{k} \in S \backslash T$ for all $k \geq 1$. Since $S \backslash T$ is finite, there exist $k, n \geq 1$ such that $f y^{\prime}(\gamma \alpha)^{k}=f y^{\prime}(\gamma \alpha)^{k+n}$. Hence,

$$
f y^{\prime}(\gamma \alpha)^{k}=f y^{\prime}(\gamma \alpha)^{k+n r}
$$


for all $r \geq 1$. Since $y=x f$, we have $y y^{\prime}(\gamma \alpha)^{k}=y y^{\prime}(\gamma \alpha)^{k+n r}$ for all $r \geq 1$. Now

$$
y=y y^{\prime} y=y y^{\prime}(\gamma \alpha)^{k} y(\beta \delta)^{k}=y y^{\prime}(\gamma \alpha)^{k+n r} y(\beta \delta)^{k}=y y^{\prime}(\gamma \alpha)^{n r} y
$$

for all $r \geq 1$. Hence, we may assume that $y^{\prime}(\gamma \alpha)^{n r} \in S \backslash T$ for all $r \geq 1$ (otherwise the assertion follows by subcase $2 \mathrm{~b}$ and we obtain that $x \mathcal{D}^{T} y$ ). So, since $S \backslash T$ is finite, there exists $r_{1}<r_{2}$ with $r_{2}-r_{1}>1$ such that $y^{\prime}(\gamma \alpha)^{n r_{1}}=y^{\prime}(\gamma \alpha)^{n r_{2}}$. Then

$$
\begin{aligned}
y^{\prime} y(\beta \delta)^{n} & =y^{\prime}(\gamma \alpha)^{n r_{1}} \cdot y(\beta \delta)^{n\left(r_{1}+1\right)}=y^{\prime}(\gamma \alpha)^{n r_{2}} \cdot y(\beta \delta)^{n\left(r_{1}+1\right)} \\
& =y^{\prime}(\gamma \alpha)^{n\left(r_{2}-r_{1}-1\right)} y .
\end{aligned}
$$

Combining (8) and (9) yields

$$
y=y \cdot y^{\prime}(\gamma \alpha)^{n\left(r_{2}-r_{1}-1\right)} y=y y^{\prime} y \cdot(\beta \delta)^{n}=y(\beta \delta)^{n}
$$

and so $x \mathcal{D}^{T} y$ by Lemma 4.10 .

Case 3. $x=y f$ and $y=x t$ for some $t \in T$ and $f \in S \backslash T$. This case is similar to case 2 .

Case 4. $x=y f_{1}$ and $y=x f_{2}$ for some $f_{1}, f_{2} \in S \backslash T$. Once again we will distinguish the following three subcases:

Subcase 4a. $x^{\prime} \in T$ and $y^{\prime} \in T$. Observe that $x x^{\prime} \mathcal{R}^{S} y y^{\prime}$, so that $x x^{\prime} \mathcal{R}^{T} y y^{\prime}$, by properties of idempotents, and it follows immediately that $x \mathcal{R}^{T} y$.

Subcase 4b. $x^{\prime} \in S \backslash T$ and $y^{\prime} \in T$. First note that

$$
x=y y^{\prime} \cdot y f_{1}, y y^{\prime}=x \cdot f_{2} y^{\prime} .
$$

If either of $y f_{1}$ or $f_{2} y^{\prime}$ is in $T$, then $x \mathcal{D}^{T} y y^{\prime}$ by cases $1-3$, and we are done. Since $y \mathcal{R}^{T} y y^{\prime}$, then $x \mathcal{D}^{T} y$ and we are done. Hence, $y f_{1} \in S \backslash T$ and $f_{2} y^{\prime} \in S \backslash T$, and without loss of generality we may assume that $y^{2}=y$ and $y^{\prime}=y$. Then $y=x \cdot f_{2} y$. If $f_{2} y \in T$ then we reduce to case 3 and the proof is complete. So we may assume that $f_{2} y \in S \backslash T$. Then, as before, $f_{2}(\gamma \alpha)^{k} \in S \backslash T$ for all $k \geq 1$. Then $f_{2}(\gamma \alpha)^{k+n}=f_{2}(\gamma \alpha)^{k}$ for some $k, n \geq 1$. This implies $f_{2} y(\beta \delta)^{n}=f_{2} y$ and so

$$
y=x f_{2} y=x f_{2} y(\beta \delta)^{n}=y(\beta \delta)^{n} .
$$

Then by Lemma $4.10, x \mathcal{D}^{T} y$.

Subcase 4c. $y^{\prime} \in S \backslash T$. Then in the same way as in case 2c one can show that this subcase can be reduced to subcase $4 \mathrm{~b}$ or to case 3 .

5. Finitely many ideals. In Gray and Ruškuc [10] it was proved that if $T$ is a subsemigroup of finite Green index in a semigroup $S$, then $T$ has finitely many right (respectively left) ideals if and only if $S$ has finitely many right (resp. left) ideals. In this section we prove the corresponding theorem for the case of two-sided ideals. In particular, this provides a positive solution to Ruškuc [23, Open Problem 11.3 (i)].

THEOREM 5.1. Let $S$ be a semigroup and let $T$ be a subsemigroup of $S$ with finite Green index. Then $T$ has finitely many ideals if and only if $S$ has finitely many ideals. 
Proof. As usual, we assume without loss of generality that $S$ has an identity element and that $1 \in T$.

$(\Rightarrow$ ) Suppose that $T$ has finitely many ideals, or, equivalently, finitely many $\mathcal{J}$ classes. Let $J^{S}$ be an arbitrary $\mathcal{J}$-class of $S$. Then $J^{S} \cap T$ is a union of $\mathcal{J}$-classes of $T$, while $J^{S} \cap(S \backslash T)$ is a union of relative $\mathcal{R}^{T}$-classes of $S$. It follows that $S$ has finitely many $\mathcal{J}$-classes.

$(\Leftarrow)$ Let now $S$ have finitely many ideals, and suppose that $T$ has infinitely many ideals. Then there exists a $\mathcal{J}$-class $J_{u}^{S}$ of $S$ which contains infinitely many $\mathcal{J}$-classes of $T$. In particular, $J_{u}^{S}$ either contains an infinite chain or an infinite anti-chain of $\mathcal{J}$-classes of $T$. In either case, for an arbitrary $N \in \mathbb{N}$ we can pick $u_{1}, \ldots, u_{N} \in J_{u}^{S} \cap T$ such that

$$
J_{u_{i}}^{T} \not \leq J_{u_{j}}^{T}(1 \leq i<j \leq N) .
$$

We choose a specific $N$ as follows. Let $P+1$ be the Green index of $T$ in $S$; thus, $P$ is equal to the number of $\mathcal{H}^{T}$-classes in $S \backslash T$. Let $Q=P^{2}+2$, and let $N$ be the Ramsey number $R(Q, Q, Q)$. Recall that this means that for every edge colouring of the complete graph of size $N$ with three colours there exists a monochromatic complete subgraph with $Q$ vertices.

Since $u_{1}, \ldots, u_{N}$ are all $\mathcal{J}$-related in $S$, we can write

$$
u_{i}=\alpha_{i} u_{i+1} \beta_{i}\left(i=1, \ldots, N-1 ; \alpha_{i}, \beta_{i} \in S\right) .
$$

Define

$$
\alpha_{i, j}=\alpha_{i} \alpha_{i+1} \ldots \alpha_{j-1}, \beta_{i, j}=\beta_{j-1} \ldots \beta_{i+1} \beta_{i}(1 \leq i<j \leq N)
$$

These elements satisfy

$$
u_{i}=\alpha_{i, j} u_{j} \beta_{i, j}(1 \leq i<j \leq N) .
$$

From (13) and (10) it follows that for all $1 \leq i<j \leq N$ at least one of $\alpha_{i, j}, \beta_{i, j}$ is not in $T$. Recalling $N=R(Q, Q, Q)$, it follows by Ramsey's theorem that there exists a set $I \subseteq\{1, \ldots, N\}$ of size $Q$ such that one of the following three possibilities holds:

$$
\begin{aligned}
& \alpha_{i, j}, \beta_{i, j} \in S \backslash T(i, j \in I, i<j), \\
& \alpha_{i, j} \in S \backslash T, \beta_{i, j} \in T(i, j \in I, i<j), \\
& \alpha_{i, j} \in T, \beta_{i, j} \in S \backslash T(i, j \in I, i<j) .
\end{aligned}
$$

Furthermore, by discarding the elements of $\{1, \ldots, N\}$ that do not belong to $I$, and re-indexing, we may take

$$
I=\{1, \ldots, Q\}
$$

First suppose that (14) holds. Each of the $Q-1=P^{2}+1$ pairs $\left(\alpha_{i, Q}, \beta_{i, Q}\right)(1 \leq i<$ $Q)$ belongs to $(S \backslash T) \times(S \backslash T)$. Since the number of $\mathcal{H}^{T}$-classes in $S \backslash T$ is precisely $P$, it follows by the Pigeonhole Principle that for some $1 \leq i<j<Q$ we have

$$
\left(\alpha_{i, Q}, \alpha_{j, Q}\right),\left(\beta_{i, Q}, \beta_{j, Q}\right) \in \mathcal{H}^{T},
$$


and write

$$
\alpha_{i, Q}=a \alpha_{j, Q}, \beta_{i, Q}=\beta_{j, Q} b(a, b \in T) .
$$

Now we have

$$
\begin{aligned}
& u_{i}=\alpha_{i, Q} u_{Q} \beta_{i, Q} \\
& =a \alpha_{j, Q} u_{Q} \beta_{j, Q} b \text { (by (18)) } \\
& =a u_{j} b \quad \text { (by (13)), }
\end{aligned}
$$

contradicting (10).

Now suppose that (15) holds. Again, using the Pigeonhole Principle, this time applied to $Q-1$ elements $\alpha_{i, Q} \in S \backslash T(1 \leq i<Q)$, we see that there exist $i, j(1 \leq i<$ $j<Q$ ) such that

$$
\left(\alpha_{i, Q}, \alpha_{j, Q}\right) \in \mathcal{H}^{T}
$$

Let $a \in T$ be such that

$$
\alpha_{i, Q}=a \alpha_{j, Q}
$$

Now we have

$$
\begin{array}{rlr}
u_{i} & =\alpha_{i, Q} u_{Q} \beta_{i, Q} & (\text { by (13)) } \\
& =a \alpha_{j, Q} u_{Q} \beta_{i, Q} & (\text { by (20)) } \\
& =a \alpha_{j, Q} u_{Q} \beta_{j, Q} \beta_{i, j} & (\text { by (12)) } \\
& =a u_{j} \beta_{i, j} & (\text { by (13)). }
\end{array}
$$

But from (15) we have $\beta_{i, j} \in T$, and this contradicts (10). Case (16) can be eliminated by a dual argument, and the theorem is proved.

6. Minimal conditions for ideals. Recall that a semigroup $S$ is said to have property $\min _{R}\left(\right.$ respectively $\left.\min _{J}\right)$ if every descending chain $R_{x_{1}}^{S} \geq R_{x_{2}}^{S} \geq R_{x_{3}}^{S} \geq \cdots$ (respectively $J_{x_{1}}^{S} \geq J_{x_{2}}^{S} \geq J_{x_{3}}^{S} \geq \cdots$ ) of $\mathcal{R}$ - (respectively $\mathcal{J}$-) classes of $S$ eventually stabilizes. Obviously, $\min _{R}$ and $\min _{J}$ are finiteness conditions.

THEOREM 6.1. Let $S$ be a semigroup and let $T$ be a subsemigroup of $S$ with finite Green index. Then $T$ satisfies $\min _{R}$ if and only if $S$ satisfies $\min _{R}$.

Proof. Without loss of generality we may assume that $S$ has an identity 1 and $1 \in T$.

$(\Rightarrow)$ Suppose $T$ satisfies $\min _{R}$, but that in $S$ we have an infinite decreasing chain $R_{x_{1}}^{S}>R_{x_{2}}^{S}>R_{x_{3}}^{S}>\cdots$ of $\mathcal{R}$-classes.

If there are infinitely many elements from $S \backslash T$ among $x_{1}, x_{2}, \ldots$, then there exists $i<j$ such that $x_{i} \mathcal{R}^{T} x_{j}$, implying $x_{i} \mathcal{R}^{S} x_{j}$, a contradiction. Hence, there are only finitely many $i$ such that $x_{i} \in S \backslash T$, and without loss of generality we may assume that in fact $x_{i} \in T$ for all $i \geq 1$. Now for every $n \geq 2$ there exists $p_{n} \in S$ such that $x_{n-1} p_{n}=x_{n}$. Then $x_{1} \cdot p_{2} \cdots p_{i}=x_{i}$ for all $i \geq 2$. If $p_{2} \cdots p_{i} \in S \backslash T$ for all $i \geq 2$, then, since there are finitely many $\mathcal{R}^{T}$-classes in $S \backslash T$, there would exist $1<i<j$ such that 
$p_{2} \cdots p_{i} \mathcal{R}^{T} p_{2} \cdots p_{j}$ and so $x_{1}=x_{1} p_{2} \cdots p_{i} \mathcal{R}^{S} x_{1} p_{2} \cdots p_{j}=x_{j}$, a contradiction. Hence, there exists $i_{2}>i_{1}=1$ such that $p_{2} \cdots p_{i_{2}} \in T$. Then $R_{x_{i_{1}}}^{T} \geq R_{x_{i_{2}}}^{T}$. Analogously, there exists $i_{3}>i_{2}$ such that $R_{x_{i_{2}}}^{T} \geq R_{x_{i_{3}}}^{T}$. Proceeding in this way, there exists an infinite sequence $i_{1}<i_{2}<i_{3}<\cdots$ such that $R_{x_{i_{1}}}^{T} \geq R_{x_{i_{2}}}^{T} \geq R_{x_{i_{3}}}^{T} \geq \cdots$. Since every $x_{i}$ lies in $T$ and $T$ satisfies $\min _{R}$, we must have $R_{x_{i_{k}}}^{T}=R_{x_{i_{k+1}}}^{T}$ for some $k$. Then $R_{x_{i_{k}}}^{S}=R_{x_{i_{k+1}}}^{S}$, a contradiction.

$(\Leftarrow)$ Suppose $S$ satisfies $\min _{R}$, but that in $T$ we have an infinite descending chain $R_{x_{1}}^{T}>R_{x_{2}}^{T}>R_{x_{3}}^{T}>\cdots$ where $x_{i} \in T$. Since $R_{x_{1}}^{S} \geq R_{x_{2}}^{S} \geq R_{x_{3}}^{S} \geq \cdots$, we may assume without loss of generality that $R_{x_{n}}^{S}=R_{x_{n+1}}^{S}$ for all $n \geq 1$. Then for every $n \geq 1$ there exists $q_{n} \in S$ with $x_{n+1} q_{n}=x_{n}$. Now

$$
x_{i}=x_{i+1} q_{i}=\cdots=x_{n+1} q_{n} \cdots q_{i}
$$

for all $1 \leq i \leq n$. Hence, $q_{n} \cdots q_{i} \in S \backslash T$ for all $1 \leq i \leq n$. Then there exist numbers $i<$ $j<N$ such that $q_{N} \cdots q_{i} \mathcal{H}^{T} q_{N} \cdots q_{j}$. In particular, there exists $t \in T$ with $q_{N} \cdots q_{i}=$ $q_{N} \cdots q_{j} \cdot t$. Then

$$
x_{i}=x_{N+1} q_{N} \cdots q_{i}=x_{N+1} q_{N} \cdots q_{j} t=x_{j} t
$$

a contradiction.

REMARK 6.2. The above proof does not use the full strength of the assumption that $T$ has finite Green index in $S$, i.e. that the number of $\mathcal{H}^{T}$-classes in $S \backslash T$ is finite, but only that there are finitely many $\mathcal{R}^{T}$-classes in $S \backslash T$.

Now we will prove an analogue of Theorem 6.1 for $\min _{J}$. For this we will require the following lemma.

LEMMA 6.3. Let T be a subsemigroup of finite Green index in a semigroup $S$. Let also $J_{x_{1}}^{S}>J_{x_{2}}^{S}>J_{x_{3}}^{S}>\cdots$ be an infinite descending chain of $\mathcal{J}$-classes of $S$ where $x_{i} \in T$ for all $i \geq 1$. Then there is a sequence $n_{1}<n_{2}<n_{3}<\cdots$ such that $J_{x_{n_{1}}}^{T} \geq J_{x_{n_{2}}}^{T} \geq J_{n_{n_{3}}}^{T} \geq \cdots$.

Proof. For each $n \geq 1$ there exist $p_{n}, q_{n} \in S$ such that $x_{n+1}=p_{n} x_{n} q_{n}$. Define $p_{i, j}=$ $p_{j-1} \cdots p_{i}$ and $q_{i, j}=q_{i} \cdots q_{j-1}$ for all $1 \leq i<j$. Then $x_{j}=p_{i, j} x_{i} q_{i, j}$ for all $1 \leq i<j$. By Ramsey's theorem there exists an infinite subset $I \subseteq \mathbb{N}$ such that $p_{i, j} \in T$ for all $i, j \in I$ with $i<j$, or $p_{i, j} \in S \backslash T$ for all $i, j \in I$ with $i<j$; and $q_{i, j} \in T$ for all $i, j \in I$ with $i<j$, or $q_{i, j} \in S \backslash T$ for all $i, j \in I$ with $i<j$. By renumbering, without loss of generality we may assume that $I=\mathbb{N}$. If all $p_{i, j}$ and $q_{i, j}$ are from $T$, then $J_{x_{1}}^{T} \geq J_{x_{2}}^{T} \geq J_{x_{3}}^{T} \geq \cdots$ and we are done. Hence, suppose that all $p_{i, j}$ are from $S \backslash T$ (the case when all $q_{i, j}$ are from $S \backslash T$ being analogous). Now consider two possible cases:

Case 1. $q_{i, j} \in T$ for all $1 \leq i<j$. By Ramsey's theorem there exists an infinite subset $J \subseteq \mathbb{N}$ such that all the $p_{i, j}$ with $i, j \in J$ and $i<j$ lie in the same $\mathcal{H}^{T}$ class. After renumbering we may assume that $J=\mathbb{N}$. Then, in particular, $p_{n+1} p_{n}=$ $p_{n, n+2} \mathcal{H}^{T} p_{n, n+1}=p_{n}$ for all $n \geq 1$. Hence, there exists $t_{n+1} \in T$ such that $p_{n+1} p_{n}=$ $t_{n+1} p_{n}$. Then

$$
x_{n+2}=p_{n+1} p_{n} x_{n} q_{n} q_{n+1}=t_{n+1} p_{n} x_{n} q_{n} q_{n+1}=t_{n+1} x_{n+1} q_{n+1} \in T x_{n+1} T
$$

for all $n \geq 1$. Therefore, $J_{x_{2}}^{T} \geq J_{x_{3}}^{T} \geq J_{x_{4}}^{T} \geq \cdots$. 
Case 2. $q_{i, j} \in S \backslash T$ for all $1 \leq i<j$. By the Pigeonhole Principle there exist numbers $N<i<j$ such that $p_{N, i} \overline{\mathcal{H}}^{T} p_{N, j}$ and $q_{N, i} \mathcal{H}^{T} q_{N, j}$. Then there exist $t_{1}, t_{2} \in T$ such that $p_{N, i}=t_{1} p_{N, j}$ and $q_{N, i}=q_{N, j} t_{2}$. Then

$$
x_{i}=p_{N, i} x_{N} q_{N, i}=t_{1} p_{N, j} x_{N} q_{N, j} t_{2}=t_{1} x_{j} t_{2},
$$

and so $J_{x_{i}}^{S}=J_{x_{j}}^{S}$, a contradiction.

THEOREM 6.4. Let $S$ be a semigroup and let $T$ be a subsemigroup of $S$ with finite Green index. Then $T$ satisfies $\min _{J}$ if and only if $S$ satisfies $\min _{J}$.

Proof. Without loss we may assume that $S$ has an identity 1 and that $1 \in T$.

$(\Rightarrow)$ Suppose $T$ satisfies $\min _{J}$, but in $S$ we have $J_{x_{1}}^{S}>J_{x_{2}}^{S}>J_{x_{3}}^{S}>\cdots$ for some $x_{i} \in S$. As in the proof of Theorem 6.1, we may assume that $x_{i} \in T$ for all $i \geq 1$. By Lemma 6.3 there exists a sequence $n_{1}<n_{2}<n_{3}<\cdots$ such that $J_{x_{n_{1}}}^{T} \geq J_{x_{n_{2}}}^{T} \geq J_{x_{n_{3}}}^{T} \geq$ $\cdots$. Therefore, $J_{x_{k}}^{T}=J_{x_{k+1}}^{T}$ for some $k$. Then $J_{x_{k}}^{S}=J_{x_{k+1}}^{S}$, a contradiction.

$(\Leftarrow)$ Suppose $S$ satisfies $\min _{J}$, but $J_{x_{1}}^{T}>J_{x_{2}}^{T}>J_{x_{3}}^{T}>\cdots$ for some $x_{i} \in T$. As in

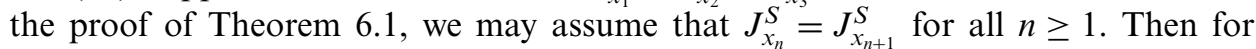
each $n \geq 1$ there exist $p_{n}, q_{n} \in S$ such that $x_{n}=p_{n} x_{n+1} q_{n}$. Define $p_{i, j}=p_{i} \cdots p_{j-1}$ and $q_{i, j}=q_{j-1} \cdots q_{i}$ for all $1 \leq i<j$. Then $x_{i}=p_{i, j} x_{j} q_{i, j}$ for all $1 \leq i<j$. It follows that for every $i<j$, either $p_{i, j} \in S \backslash T$, or $q_{i, j} \in S \backslash T$. By Ramsey's theorem and up to renumbering, we may assume that $p_{i, j} \in S \backslash T$ for all $i<j$. Furthermore, we may even assume that all of $p_{i, j}$ lie in the same $\mathcal{H}^{T}$-class.

Take arbitrary $i<j$. Then $p_{i, j} p_{j}=p_{i, j+1} \mathcal{H}^{T} p_{j, j+1}=p_{j}$ and so there exists $t \in T$ such that $p_{i, j} p_{j}=t p_{j}$. Then $x_{i}=p_{i, j} p_{j} x_{j+1} q_{j} q_{i, j}=t p_{j} x_{j+1} q_{j} q_{i, j}=t x_{j} q_{i, j}$ and so $q_{i, j} \in S \backslash T$.

Now by the Pigeonhole Principle there exist numbers $i<j<N$ such that $p_{i, N} \mathcal{H}^{T} p_{j, N}$ and $q_{i, N} \mathcal{H}^{T} q_{j, N}$. Therefore, there exist $t_{1}, t_{2} \in T$ such that $p_{i, N}=t_{1} p_{j, N}$ and $q_{i, N}=q_{j, N} t_{2}$. Then

$$
x_{i}=p_{i, N} x_{N} q_{i, N}=t_{1} p_{j, N} x_{N} q_{j, N} t_{2}=t_{1} x_{j} t_{2},
$$

a contradiction. This proves the theorem.

Another natural finiteness condition, related to (and weaker than) $\min _{J}$ is that of having a minimal two-sided ideal. The following result is easy to prove, but we include it for completeness.

Proposition 6.5. Let $T$ be a subsemigroup of finite Green index in a semigroup $S$. If $T$ has a minimal ideal, then $S$ has a minimal ideal.

Proof. Let $I$ be a minimal ideal in $T$ and assume that $S$ does not have a minimal ideal. Take any $x \in I$. Then there exists an infinite chain $J_{x}^{S}>J_{x_{1}}^{S}>J_{x_{2}}^{S}>\cdots$ where $x_{i} \in S$. As in the proof of Theorem 6.1, we may assume that $x_{i} \in T$ for all $T$. Now $J_{x_{1}}^{T} \geq J_{x}^{T}$ and so $J_{x_{1}}^{S} \geq J_{x}^{S}$, a contradiction.

The converse of Proposition 6.5 does not hold. Indeed, if $T$ is any semigroup, the semigroup $S=T^{0}$, obtained by adjoining a zero element to $T$, has $T$ as a subsemigroup of finite Green (and indeed Rees) index, and has $\{0\}$ as its minimal ideal.

7. All ideals have finite Rees index. In this section we present a result which gives a positive answer to [23, Open Problem 11.3 (ii)]. 
THEOREM 7.1. Let $S$ be a semigroup and let $T$ be a subsemigroup of $S$ with finite Rees index. If every ideal in $S$ has finite Rees index, then every ideal in T has finite Rees index.

Proof. Suppose that every ideal in $S$ has finite Rees index. Let $I$ be any ideal in $T$, and set $F=S \backslash T$. For $i \in I$ define two sets

$$
X_{i}=\{f \in F: f i \in I\}, \quad Y_{i}=\{f \in F: \text { if } \in I\} .
$$

Let $i_{1}$ (resp. $i_{2}$ ) be any element of $I$ such that the set $X_{i_{1}}$ (resp. $Y_{i_{2}}$ ) has the maximal possible size.

We claim that

$$
F i_{1} I \subseteq F \cup I, I i_{2} F \subseteq F \cup I .
$$

It suffices to prove the first inclusion; the second is dual. Suppose that there exist $f \in F$, $j \in I$ such that $f i_{1} j \in T \backslash I$. Then $f \notin X_{i_{1}}$, and for any $j_{1} \in I$ we have $f i_{1} j_{1} \in I$. This implies $X_{i_{1} \ddot{j}_{1}} \supseteq X_{i_{1}} \cup\{f\}$, contradicting the choice of $i_{1}$.

Now consider the ideal $J=S^{1} i_{1} i_{2} S^{1}$ of $S$. We have

$$
\begin{aligned}
S^{1} i_{1} i_{2} S^{1} & =\left(F \cup T^{1}\right) i_{1} i_{2}\left(F \cup T^{1}\right)=F i_{1} i_{2} F \cup T^{1} i_{1} i_{2} F \cup F i_{1} i_{2} T^{1} \cup T^{1} i_{1} i_{2} T^{1} \\
& \subseteq F i_{1} i_{2} F \cup I i_{2} F \cup F i_{1} I \cup I I \subseteq F i_{1} i_{2} F \cup I \cup F .
\end{aligned}
$$

Note that the set $F i_{1} i_{2} F$ is finite, and so $I \cap J$ has finite index in $J$. By assumption $J$ has finite Rees index in $S$. It follows that $I$ has finite Rees index in $S$, and hence in $T$ as well.

REMARK 7.2. The converse of Theorem 7.1 does not hold: Adjoining a zero to any infinite semigroup $S$ results in a semigroup with an ideal (namely $\{0\}$ ) of infinite Rees index. Thus, a counterexample to the converse of Theorem 7.1 may be obtained by taking an infinite semigroup whose ideals all have finite Rees index (e.g. an infinite group) and adjoining a zero.

The analogue of Theorem 7.1 for right ideals also holds: Assume that $T$ is a subsemigroup of finite Rees index in $S$ and that every right ideal in $S$ has finite Rees index in $S$. Let $R$ be a right ideal in $T$. Take any $r \in R$. Then $r S=r T \cup r(S \backslash T) \subseteq$ $R \cup r(S \backslash T)$ and the complement of $r S$ in $S$ must be finite. Hence, $T \backslash R$ is finite and so $R$ has finite Rees index in $T$.

Question 7.3. Can the assumption of $T$ having finite Rees index in Theorem 7.1 be weakened to finite Green index? In other words: if every ideal of $S$ has finite Rees index, and if $T$ is a subsemigroup of $S$ of finite Green index, is it necessarily the case that every ideal of $T$ has finite Rees index?

To finish off this section, we prove the following proposition about the related finiteness condition of every subsemigroup having finite Green index.

Proposition 7.4. If every subsemigroup of a semigroup $S$ has finite Green index in $S$, then $S$ is finite.

Proof. Take any element $a \in S$ and let $T=\langle a\rangle$. If $T$ is finite, then $S$ is finite too, since there are finitely many $\mathcal{R}^{T}$-classes and each is bounded in size by $T$. Now suppose that $T$ is infinite. Consider the subsemigroup $T^{\prime}=\left\langle a^{2}\right\rangle$. Since $T^{\prime}$ has finite Green index 
in $S$, we obtain that $a^{2 k+1} \mathcal{H}^{T} a^{2 n+1}$ for some $1 \leq k<n$. Then $a^{2 k+1}=a^{2 n+1} \cdot a^{2 m}$ for some $m \geq 0$, a contradiction.

8. Global torsion. For a semigroup $S$ and $n \in \mathbb{N}$ define $S^{n}=\left\{s_{1} \cdots s_{n}\right.$ : $\left.s_{1}, \ldots, s_{n} \in S\right\}$. We say that $S$ has global torsion if $S^{n+1}=S^{n}$ for some $n \in \mathbb{N}$. It is clear that $S \supseteq S^{2} \supseteq S^{3} \supseteq \ldots$, and it follows that global torsion is a finiteness condition.

THEOREM 8.1. Let $S$ be a semigroup and let $T$ be a subsemigroup of $S$ with finite Green index. If T has global torsion, then $S$ has global torsion as well.

Proof. Let $m \in \mathbb{N}$ such that $T^{m+1}=T^{m}$, and let $r$ be the number of $\mathcal{H}^{T}$-classes in $S \backslash T$. We begin by proving the following.

LEMMA 8.2. For any $s_{1}, \ldots, s_{r+1} \in S$ we either have $s_{1} \cdots s_{r+1} \in S^{r+2}$ or else $s_{1} \cdots s_{i} \in T$ for some $i \in\{1, \ldots, r+1\}$.

Proof. Assume that $s_{1} \cdots s_{i} \in S \backslash T$ for all $1 \leq i \leq r+1$. Then there exist $1 \leq i<$ $j \leq r+1$ such that $s_{1} \cdots s_{i} \mathcal{H}^{T} s_{1} \cdots s_{j}$. Hence, there exists $t \in T^{1}$ such that

$$
s_{1} \cdots s_{i}=\left(s_{1} \cdots s_{i}\right)\left(s_{i+1} \cdots s_{j} t\right) \in S^{i+1} .
$$

Hence,

$$
s_{1} \ldots s_{r+1}=\left(s_{1} \ldots s_{i}\right) s_{i+1} \ldots s_{r+1} \in S^{i+1} s_{i+1} \ldots s_{r+1} \subseteq S^{r+2},
$$

as required.

Resuming the proof of the theorem, let $n=(r+1) m$. We claim that $S^{n+1}=S^{n}$. Clearly, $S^{n+1} \subseteq S^{n}$. Let $s_{1}, \ldots, s_{n} \in S$ be arbitrary so that $s_{1} \cdots s_{n}$ is a typical element of $S^{n}$. If for any $i \in\{1, \ldots, n-r\}$ we have $s_{i} s_{i+1} \cdots s_{i+r} \in S^{r+2}$ then we also have $s_{1} s_{2} \ldots s_{n} \in S^{n+1}$ and the proof is finished. The alternative is, by Lemma 8.2, that for every $i \in\{1, \ldots, n-r\}$ there exists $j \in\{0, \ldots, r\}$ such that $s_{i} s_{i+1} \cdots s_{i+j} \in T$. In particular, there exist $j_{1}<j_{2}<\cdots<j_{m}$ belonging to $\{1, \ldots, n\}$ such that $j_{k+1}-j_{k} \leq$ $r+1$ for all $k=1, \ldots, m-1$ and

$$
s_{1} s_{2} \cdots s_{j_{1}}, s_{j_{1}+1} s_{j_{1}+2} \cdots s_{j_{2}}, \ldots, s_{j_{m-1}+1} s_{j_{m-1}+2} \ldots s_{j_{m}} \in T
$$

But then

$$
s_{1} s_{2} \cdots s_{j_{m}} \in T^{m}=T^{m+1}=\cdots=T^{j_{m}}=T^{j_{m}+1} \subseteq S^{j_{m}+1} .
$$

Abbreviating $j_{m}=j$, we now have

$$
s_{1} \ldots s_{n}=\left(s_{1} \ldots s_{j}\right)\left(s_{j+1} \ldots s_{n}\right) \in S^{j+1} s_{j+1} \ldots s_{n} \subseteq S^{n+1},
$$

completing the proof.

REMARK 8.3. The converse of Theorem 8.1 does not hold: Adjoining an identity element to an arbitrary semigroup $T$ yields a semigroup $S$ such that $S^{2}=S$.

9. Eventual regularity. We close the paper by discussing one more important finiteness condition, this time not related to ideals. 
Definition 9.1. A semigroup $S$ is eventually regular if for every $s \in S$ there exists $n \in \mathbb{N}$ such that $s^{n}$ is a regular element of $S$.

The class of eventually regular semigroups (also called $\pi$-regular) was introduced by Edwards in [8]. Further results on these semigroups include [1, 7, 11]. Clearly, every finite semigroup is eventually regular, i.e. eventual regularity is a finiteness condition.

THEOREM 9.2. Let $S$ be a semigroup and let $T$ be a subsemigroup with finite Green index. Then $S$ is eventually regular if and only if $T$ is eventually regular.

Proof. Suppose that $T$ is eventually regular and let $s \in S$ be arbitrary. If $s^{m} \in T$ for some $m \in \mathbb{N}$, then, since $T$ is eventually regular, $\left(s^{m}\right)^{n}=s^{m n}$ is regular in $T$ (and hence also in $S$ ) for some $n \in \mathbb{N}$. Otherwise $s^{m} \notin T$ for all $m$ and since $T$ has finite Green index in $S$ there exist $n, r \in \mathbb{N}$ with $s^{n+r} \mathcal{H}^{T} s^{n}$. Then as in the proof of Gray and Ruškuc [10, Theorem 18] choosing $z \in \mathbb{N}$ with $0 \leq z \leq r-1$ and $n+z \equiv 0(\bmod r)$ we have $\left(s^{n+z}\right)^{2} \mathcal{H}^{T} s^{n+z}$. By Proposition 2.1 (ii) we have that the relative $\mathcal{H}^{T}$ class of $s^{n+z}$ is a group, and hence $s^{n+z}$ is a regular element.

For the converse, suppose that $S$ is eventually regular and let $t \in T$. Since $S$ is eventually regular, there exists an infinite subset $I \subseteq \mathbb{N}$ such that $t^{i}$ is regular in $S$ for all $i \in I$. For each $i \in I$, let $s_{i}$ be an inverse of $t^{i}$ in $S$, so

$$
t^{i} s_{i} t^{i}=t^{i}, \quad s_{i} t^{i} s_{i}=s_{i}
$$

If $s_{i} \in T$ for some $i \in I$ then $t^{i}$ is regular in $T$ and we are done, so suppose otherwise. For all $i \in I$, set $f_{i}=t^{i} s_{i}$ noting that by (21), $f_{i}$ is an idempotent satisfying $f_{i} \mathcal{R}^{S} t^{i}$ and $f_{i} \mathcal{L}^{S} s_{i}$. Since $s_{i} \in S \backslash T$ for all $i \in I$, and $T$ has finite Green index in $S$, it follows that there is an infinite subset $J \subseteq I$ such that for all $i, j \in J$ we have $s_{i} \mathcal{H}^{T} s_{j}$. Let $i, j \in J$ be arbitrary, with $i<j$ say. Then

$$
f_{i} \mathcal{L}^{S}{ }_{s_{i}} \mathcal{L}^{S}{ }_{j} \mathcal{L}^{S} f_{j}
$$

and therefore $f_{i} f_{j}=f_{i}$. Since $\mathcal{R}$ on $S$ is a left congruence, $t^{j} \mathcal{R}^{S} f_{j}$ implies $f_{i} t^{j} \mathcal{R}^{S} f_{i} f_{j}$ and hence

$$
t^{j}=t^{i} t^{j-i}=t^{i} s_{i} t^{i} t^{j-i}=\left(t^{i} s_{i}\right) t^{j}=f_{i} t^{j} \mathcal{R}^{S} f_{i} f_{j}=f_{i} \mathcal{R}^{S} t^{i}
$$

By a dual argument $t^{j} \mathcal{L}^{S} t^{i}$ and hence $t^{j} \mathcal{H}^{S} t^{i}$.

Since $i, j \in J$ were arbitrary, it follows that $t^{k} \mathcal{H}^{S} t^{l}$ for all $k, l \in J$. By Gray and Ruškuc [10, Proposition 10] each $\mathcal{H}^{S}$-class of $S$ is a union of finitely many $\mathcal{H}^{T}$-classes. Since $J$ is infinite, it follows that there exist distinct $p, q \in J$ with $t^{p} \mathcal{H}^{T} t^{q}$. Now as in the proof of the above converse we can find a number $y \in \mathbb{N}$ with $\left(t^{y}\right)^{2} \mathcal{H}^{T} t^{y}$, and we conclude that $t^{y}$ is a regular element of $T$.

Acknowledgements. R. Gray was supported by an EPSRC post-doctoral fellowship, and partially supported by FCT and FEDER, project POCTI-ISFL1-143 of Centro de Álgebra da Universidade de Lisboa, and by the project PTDC/MAT/69514/2006. 


\section{REFERENCES}

1. K. Auinger and T. E. Hall, Representations of semigroups by transformations and the congruence lattice of an eventually regular semigroup. Int. J. Algebra Comput. 6(1996), 655-685.

2. J.-C. Birget, The synthesis theorem for finite regular semigroups, and its generalization. J. Pure Appl. Algebra 55 (1988), 1-79.

3. R. V. Book and F. Otto, String-rewriting systems, Texts and Monographs in Computer Science (Springer-Verlag, New York, NY, 1993).

4. A. J. Cain, R. Gray and N. Ruškuc, Green index in semigroup theory: Generators, presentations, and automatic structures, Semigroup Forum 85(3) (2012), 448-476. doi:10.1007/s00233-012-9406-2.

5. A. J. Cain, E. F. Robertson and N. Ruškuc, Cancellative and Malcev presentations for finite Rees index subsemigroups and extensions, J. Austral. Math. Soc. 84 (2008), 39-61.

6. D. Day, Expository lectures on topological semigroups, in Algebraic theory of machines, languages and semigroups (Arbib M. A., Editor) (Academic Press, Atlanta, GA, 1968), 269-296.

7. D. Easdown, Biordered sets of eventually regular semigroups, Proc. Lond. Math. Soc. 49 (1984), 483-503. 23-38.

8. P. M. Edwards, Eventually regular semigroups, Bull. Austral. Math. Soc. 28 (1983),

9. G. Z. Elston and C. L. Nehaniv, Holonomy embedding of arbitrary stable semigroups, Int. J. Algebra Comput. 12 (2002), 791-810.

10. R. Gray and N. Ruškuc, Green index and finiteness conditions for semigroups, J. Algebra 320 (2008), 3145-3164.

11. P. M. Higgins, A class of eventually regular semigroups determined by pseudo-random sets, J. Lond. Math. Soc. 48 (1993), 87-102.

12. M. Hoffmann, R. M. Thomas and N. Ruškuc, Automatic semigroups with subsemigroups of finite Rees index, Int. J. Algebra Comput. 12 (2002), 463-476.

13. J. M. Howie, Fundamentals of semigroup theory, LMS Monographs, vol. 7 (Academic Press, London, 1995).

14. A. Jura, Coset enumeration in a finitely presented semigroup, Canad. Math. Bull. 21 (1978), 37-46.

15. A. Jura, Determining ideals of a given finite index in a finitely presented semigroup, Demonstratio Math. 11 (1978), 813-827.

16. A. Jura, Some remarks on nonexistence of an algorithm for finding all ideals of a given finite index in a finitely presented semigroup, Demonstratio Math. 13 (1980), 573-578.

17. M. Kambites, Presentations for semigroups and semigroupoids, Int. J. Algebra Comput. 15 (2005), 291-308.

18. R. J. Koch and A. D. Wallace, Stability in semigroups, Duke Math. J. 24 (1957), 193-195.

19. G. Lallement, Semigroups and combinatorial applications, Pure and Applied Mathematics (John Wiley, New York, NY, 1979).

20. A. Malheiro, On trivializers and subsemigroups, in Semigroups and formal languages (André J. M. et al., Editors) (World Scientific Publications, Hackensack, NJ, 2007), 188-204.

21. L. O'Carroll, Counterexamples in stable semigroups, Trans. Amer. Math. Soc. 146 (1969), 377-386.

22. J. Rhodes and B. Steinberg, The q-theory of finite semigroups, Springer Monographs in Mathematics (Springer, New York, NY, 2009).

23. N. Ruškuc, On large subsemigroups and finiteness conditions of semigroups, Proc. Lond. Math. Soc. 76 (1998), 383-405.

24. N. Ruškuc and R. M. Thomas, Syntactic and Rees indices of subsemigroups, J. Algebra 205 (1998), 435-450.

25. A. D. Wallace, Relative ideals in semigroups II. The relations of Green, Acta Math. Acad. Sci. Hungar. 14 (1963), 137-148.

26. J. Wang, Finite complete rewriting systems and finite derivation type for small extensions of monoids, J. Algebra 204 (1998), 493-503.

27. K. B. Wong and P. C. Wong, On finite complete rewriting systems and large subsemigroups, J. Algebra 345 (2011), 242-256. 